PROCEEDINGS OF THE

AMERICAN MATHEMATICAL SOCIETY

Volume 126, Number 5, May 1998, Pages 1315-1320

S 0002-9939(98)04196-3

\title{
A NOTE ON GREENBERG'S CONJECTURE AND THE ABC CONJECTURE
}

\author{
HUMIO ICHIMURA
}

(Communicated by William W. Adams)

\begin{abstract}
For any totally real number field $k$ and any prime number $p$ Greenberg's conjecture for $(k, p)$ asserts that the Iwasawa invariants $\lambda_{p}(k)$ and $\mu_{p}(k)$ are both zero. For a fixed real abelian field $k$, we prove that the conjecture is "affirmative" for infinitely many $p$ (which split in $k$ ) if we assume the $a b c$ conjecture for $k$.
\end{abstract}

\section{INTRODUCTION}

For a number field $k$ and a prime number $p$, let $k_{\infty} / k$ be the cyclotomic $\mathbb{Z}_{p^{-}}$ extension over $k$ with its $n$th layer $k_{n}\left(k_{0}=k\right)$. Let $A_{n}$ be the Sylow $p$-subgroup of the ideal class group of $k_{n}$ and $A_{\infty}=\varliminf_{n} A_{n}$ the projective limit w.r.t. the relative norms. We denote by $\lambda_{p}=\lambda_{p}(k)$ and $\mu_{p}=\mu_{p}(k)$ the Iwasawa $\lambda$-invariant and the $\mu$-invariant associated to $A_{\infty}$, respectively. Greenberg's conjecture for $k$ and $p$ asserts that $\lambda_{p}=\mu_{p}=0$ for any totally real number field $k$ and any $p$ (cf. [Iw], p. 316, [Gr]). It is well known that the conjecture is valid if (1) there is only one prime ideal of $k$ over $p$ and it is totally ramified in $k_{\infty}$ and further $(2) A_{0}=\{1\}$ (cf. [W], Proposition 13.22). In particular, $\lambda_{p}(\mathbb{Q})=\mu_{p}(\mathbb{Q})=0$ for all $p$. Further, it is known that $\mu_{p}=0$ when $k$ is abelian over $\mathbb{Q}$ (cf. [FW]). But, the conjecture for general $k$ and $p$ is far from being settled in spite of the efforts of several authors (see [IS] and its references).

In this note, we consider the following subproblem: "For a fixed totally real number field $k(\neq \mathbb{Q})$, do there exist infinitely many prime numbers $p$ for which $\lambda_{p}=\mu_{p}=0$ ?" In view of the proposition in [W] cited above, we should confine ourselves to those $p$ which split in $k$. We prove that for a certain real abelian field $k$, the problem is "affirmative" if we assume the $a b c$ conjecture for $k$. Here, the $a b c$ conjecture is formulated as follows:

Conjecture (cf. $[\mathrm{V}]$, p. 84$)$. Let $K$ be a number field. For any $\varepsilon(>0)$ and any finite set $S$ of prime ideals of $K$, there exists a constant $C(>0)$ depending only

Received by the editors June 23, 1996 and, in revised form, October 30, 1996.

1991 Mathematics Subject Classification. Primary 11R23.

The author was partially supported by the Grants-in-Aid for Scientific Research, The Ministry of Education, Science and Culture, Japan. 
on $K, \varepsilon$ and $S$ such that

$$
\prod_{v} \max \left(\|a\|_{v},\|b\|_{v},\|c\|_{v}\right) \leq C\left(\prod_{\mathfrak{p} \mid a b c}{ }^{\prime} N \mathfrak{p}\right)^{1+\varepsilon}
$$

for all integers $a, b, c$ of $K$ with $a+b=c$. Here, $v$ runs over all absolute values of $K,\|*\|_{v}$ denotes the normalized valuation and $\mathfrak{p}$ runs over all prime ideals of $K$ with $\mathfrak{p} \mid a b c$ and $\mathfrak{p} \notin S$.

Now, let $k / \mathbb{Q}$ be a real abelian extension with $k \neq \mathbb{Q}$, and $\Delta=\operatorname{Gal}(k / \mathbb{Q})$. For a prime number $p$ with $p \nmid[k: \mathbb{Q}]$ and a $\mathbb{Q}_{p}$-character $\Psi$ of $\Delta$, let $\lambda_{p}(\Psi)$ and $\mu_{p}(\Psi)$ be the $\lambda$-invariant and the $\mu$-invariant associated to the $\Psi$-component $e_{\Psi} A_{\infty}$, respectively. Here, a $\mathbb{Q}_{p}$-character means a $\mathbb{Q}_{p}$-valued character of $\Delta$ defined and irreducible over $\mathbb{Q}_{p}$, and $e_{\Psi}$ is the idempotent of $\mathbb{Q}_{p}[\Delta]$ corresponding to $\Psi$, which is an element of $\mathbb{Z}_{p}[\Delta]$ as $p \nmid[k: \mathbb{Q}]$. By $[\mathrm{FW}], \mu_{p}(\Psi)=0$. We have $\lambda_{p}=\sum_{\Psi} \lambda_{p}(\Psi)$, $\Psi$ running over all $\mathbb{Q}_{p}$-characters of $\Delta$. Further, for the trivial character $\Psi_{0}$ of $\Delta$, we have $\lambda_{p}\left(\Psi_{0}\right)=0$ since $\lambda_{p}\left(\Psi_{0}\right)=\lambda_{p}(\mathbb{Q})$.

Theorem 1. Let $k / \mathbb{Q}$ be a real cyclic extension with $[k: \mathbb{Q}]$ an odd prime number. If the abc conjecture for $k$ is valid, then there exist infinitely many pairs $(p, \Psi)$ of a prime number $p($ with $p \nmid[k: \mathbb{Q}])$ and a nontrivial $\mathbb{Q}_{p}$-character $\Psi$ of $\Delta$ satisfying (I) $p$ splits in $k$ and (II) $\lambda_{p}(\Psi)=0$.

Theorem 2. Let $k / \mathbb{Q}$ be a real quadratic extension for which the norm of a fundamental unit is -1 . If the abc conjecture for $k$ is valid, then there exist infinitely many prime numbers $p$ satisfying (I) $p$ splits in $k$ and (II) $\lambda_{p}=0$.

When (i) $k / \mathbb{Q}$ is noncyclic or (ii) $k / \mathbb{Q}$ is cyclic and $[k: \mathbb{Q}]$ is a composite, an assertion similar to the above theorems holds without assuming the $a b c$ conjecture $($ see $\S 4)$.

\section{SOME LEMMAS}

First, we introduce some notation. Let $k / \mathbb{Q}$ be a real abelian extension with $k \neq$ $\mathbb{Q}, p$ an odd prime number with $p \nmid[k: \mathbb{Q}]$ and $\Psi$ a $\mathbb{Q}_{p}$-character of $\Delta=\operatorname{Gal}(k / \mathbb{Q})$. We fix $p$ and $\Psi$ in this section. Let $\psi$ be a fixed irreducible component of $\Psi$ over an algebraic closure $\overline{\mathbb{Q}}_{p}$ of $\mathbb{Q}_{p}$, and let $O=O_{\psi}$ be the subring of $\overline{\mathbb{Q}}_{p}$ generated by the values of $\psi$ over $\mathbb{Z}_{p}$. We identify the subring $e_{\Psi} \mathbb{Z}_{p}[\Delta]$ of $\mathbb{Z}_{p}[\Delta]$ with $O$ by $e_{\Psi} \sigma \leftrightarrow \psi(\sigma)(\sigma \in \Delta)$. Then, for a $\mathbb{Z}_{p}[\Delta]$-module $X$ (e.g. $\left.A_{n}, A_{\infty}\right)$, its $\Psi$-component $X(\Psi)=e_{\Psi} X$ (or $\left.X^{e_{\Psi}}\right)$ is considered as an $O$-module. Therefore, $A_{\infty}(\Psi)$ is regarded as a module over the completed group ring $\Lambda_{p, \Psi}=O\left[\left[\operatorname{Gal}\left(k_{\infty} / k\right)\right]\right]$. It is known to be torsion over $\Lambda_{p, \Psi}$ by [Iw], Theorem 5 . Let $r$ be the degree of the quotient field of $O$ over $\mathbb{Q}_{p}$. The invariant $\lambda_{p}(\Psi)$ (resp. $\mu_{p}(\Psi)$ ) mentioned in $\S 1$ is $r$ times the $\lambda$-invariant (resp. $\mu$-invariant) of the torsion $\Lambda_{p, \Psi}$-module $A_{\infty}(\Psi)$.

For a prime ideal $\mathfrak{p}$ of $k$ over $p$, let $k_{\mathfrak{p}}$ be the completion of $k$ at $\mathfrak{p}$ and $\mathcal{U}_{\mathfrak{p}}$ the group of principal units of $k_{\mathfrak{p}}$. We denote by $\mathcal{U}$ the group of semi-local units of $k$ at $p$, namely, $\mathcal{U}:=\prod_{\mathfrak{p} \mid p} \mathcal{U}_{\mathfrak{p}}, \mathfrak{p}$ running over all prime ideals of $k$ with $\mathfrak{p} \mid p$. The group $E$ of global units of $k$ is considered as a subgroup of $\prod_{\mathfrak{p} \mid p} k_{\mathfrak{p}}^{\times}$. Denote by $\mathcal{E}$ the closure of $E \cap \mathcal{U}$ in $\mathcal{U}$. The groups $\mathcal{U}$ and $\mathcal{E}$ can be regarded as $\mathbb{Z}_{p}[\Delta]$-modules in a natural way, and hence $\mathcal{U}(\Psi)$ and $\mathcal{E}(\Psi)$ are $O$-modules. 
We regard $\psi$ as a primitive Dirichlet character, and we denote its "dual" character by $\psi^{*}$. Namely, $\psi^{*}$ is the primitive Dirichlet character associated to $\omega \psi^{-1}$, where $\omega$ is the Teichmüller character $\mathbb{Z} / p \mathbb{Z} \rightarrow \mathbb{Z}_{p}$.

Lemma 1 (cf. [IS], Remark 4). If $\psi(p) \neq 1$ and $A_{0}(\Psi)=\{1\}$, then we have $\lambda_{p}(\Psi)=\mu_{p}(\Psi)=0$.

Lemma 2. Assume that $\Psi$ is nontrivial. If $A_{0}(\Psi)=\{1\}$ and $\mathcal{U}(\Psi)=\mathcal{E}(\Psi)$, then we have $\lambda_{p}(\Psi)=\mu_{p}(\Psi)=0$.

Lemma 1 is a refinement of the proposition in [W] cited in $\S 1$. Lemma 2 is already known when $k$ is a real quadratic field by [FK]. The assertion for the general case and its proof were communicated to the author by Hiroki Sumida.

Proof of Lemma 2. Let $M / k_{\infty}$ be the maximal pro- $p$ abelian extension unramified outside $p$ and $L / k_{\infty}$ the maximal unramified pro- $p$ abelian extension. Further, let $M_{0}$ be the maximal abelian extension of $k$ contained in $M$ and $K_{0}$ the Hilbert $p$-class field of $k$. The Galois groups $\operatorname{Gal}\left(M / k_{\infty}\right), \operatorname{Gal}\left(L / k_{\infty}\right)$, etc. are regarded as modules over $\mathbb{Z}_{p}[\Delta]$ in a natural way. By class field theory, $\operatorname{Gal}\left(L / k_{\infty}\right)$ is canonically isomorphic to $A_{\infty}$. Therefore, as $M \supset L$, it suffices to show that $\operatorname{Gal}\left(M / k_{\infty}\right)(\Psi)=\{1\}$. We have a canonical isomorphism $\operatorname{Gal}\left(M_{0} / K_{0}\right) \simeq \mathcal{U} / \mathcal{E}$ by class field theory (cf. [C], Theorem 1$)$. From this, we see that $\operatorname{Gal}\left(M_{0} / K_{0} k_{\infty}\right)(\Psi)$ is isomorphic to $\mathcal{U}(\Psi) / \mathcal{E}(\Psi)$ since $\operatorname{Gal}\left(M_{0} / K_{0} k_{\infty}\right)(\Psi)=\operatorname{Gal}\left(M_{0} / K_{0}\right)(\Psi)$ as $\Psi \neq \Psi_{0}$. On the other hand, $\operatorname{Gal}\left(K_{0} k_{\infty} / k_{\infty}\right)(\Psi)$ is naturally isomorphic to $A_{0}(\Psi)$. Therefore, under the assumptions of Lemma 2, we obtain $\operatorname{Gal}\left(M_{0} / k_{\infty}\right)(\Psi)=\{1\}$ and hence $\operatorname{Gal}\left(M / k_{\infty}\right)(\Psi)=\{1\}$ by Nakayama's lemma.

Lemma 3. Assume $\psi^{*}(p) \neq 1$. Let $X$ be a closed Galois submodule of $\mathcal{U}(\Psi)$ such that $u_{\mathfrak{q}} \not \equiv 1 \bmod \mathfrak{q}^{2}$ for some element $u=\left(u_{\mathfrak{p}}\right)_{\mathfrak{p} \mid p}$ in $X$ and some prime ideal $\mathfrak{q}$ with $\mathfrak{q} \mid p$. Then, we have $X=\mathcal{U}(\Psi)$.

Proof. We have $\mathcal{U}(\Psi) \simeq O$ because of $\psi^{*}(p) \neq 1$ (cf. [Gi], §2). Therefore, $X=$ $\mathcal{U}(\Psi)^{A}$ for some ideal $A$ of $O$ since $X$ is an $O$-submodule of $\mathcal{U}(\Psi)$. We have $A=p^{a} O$ for some integer $a(\geq 0)$ since the quotient field of $O$ is unramified over $\mathbb{Q}_{p}$ as $p \nmid[k: \mathbb{Q}]$. If $a \geq 1$, then we must have $u_{\mathfrak{p}} \equiv 1 \bmod \mathfrak{p}^{2}$ for all $u=\left(u_{\mathfrak{p}}\right)$ in $X$ and all $\mathfrak{p} \mid p$. Therefore, we obtain $A=O$ and $X=\mathcal{U}(\Psi)$.

The following lemma is easily proved and we do not give its proof.

Lemma 4. Let $K$ be a number field, $\mathfrak{p}$ a prime ideal of $K$ and $\alpha$ an element of $K$ relatively prime to $\mathfrak{p}$. If $\alpha^{n} \equiv 1 \bmod \mathfrak{p}$ but $\alpha^{n} \not \equiv 1 \bmod \mathfrak{p}^{2}$ for some integer $n$, then we have $\alpha^{N \mathfrak{p}-1} \not \equiv 1 \bmod \mathfrak{p}^{2}$.

\section{Proof of the theorems}

Let $k / \mathbb{Q}$ be $(\mathrm{A})$ a real cyclic extension with $[k: \mathbb{Q}]$ an odd prime number or (B) a real quadratic extension for which the norm of a fundamental unit is -1 . In the case (A), take a totally negative unit $\varepsilon$ of $k$ with $\varepsilon \neq-1$. Then, $N \varepsilon=-1$ as $[k: \mathbb{Q}]$ is odd. Here, $N$ denotes the norm map from $k$ to $\mathbb{Q}$. In the case (B), let $\varepsilon$ be a fundamental unit of $k$, for which we have $N \varepsilon=-1$ by assumption. Let $\|*\|_{i}$ $(1 \leq i \leq[k: \mathbb{Q}])$ be the real absolute values of $k$. Replacing $\varepsilon$ by $\varepsilon^{x}$ for some large odd integer $x$ if necessary, we may well assume that $\|\varepsilon\|_{i}$ is so large (resp. so small) for all $i$ with $\|\varepsilon\|_{i}>1$ (resp. $\|\varepsilon\|_{i}<1$ ) that

$$
\left|N\left(1-\varepsilon^{m}\right)\right|>\left|N\left(1-\varepsilon^{n}\right)\right| \text { when } m>n \geq 1 .
$$


Claim 1. Let $\mathfrak{p}$ be a prime ideal of $k$ with $\mathfrak{p} \nmid 2$. If $\varepsilon^{n} \equiv 1 \bmod \mathfrak{p}$ for some odd integer $n$, then $p=\mathfrak{p} \cap \mathbb{Q}$ splits completely in $k$.

Actually: Assume that $p$ does not split completely in $k$. Then, $\mathfrak{p}$ is the unique prime ideal of $k$ over $p$ since $[k: \mathbb{Q}]$ is a prime number. So, $\left(\varepsilon^{\sigma}\right)^{n} \equiv 1 \bmod \mathfrak{p}$ for all $\sigma \in \Delta$. Therefore, as $n$ is odd, $-1=(N \varepsilon)^{n} \equiv 1 \bmod \mathfrak{p}$. This contradicts $\mathfrak{p} \nmid 2$.

Now, we assume that the $a b c$ conjecture holds for $k$. Then, applying the inequality (1) for $\varepsilon^{n}+\left(1-\varepsilon^{n}\right)=1$, we see that for some constant $C_{1}$,

$$
\left|N\left(1-\varepsilon^{n}\right)\right| \leq C_{1}\left(\prod_{\mathfrak{p} \mid\left(1-\varepsilon^{n}\right)}^{\prime} N \mathfrak{p}\right)^{3 / 2}
$$

for all integers $n$. Here, $\mathfrak{p}$ runs over all prime ideals of $k$ with $\mathfrak{p} \mid\left(1-\varepsilon^{n}\right)$ and $\mathfrak{p} \nmid 2(1-\varepsilon)$. Using this inequality, we show

Claim 2. Under the $a b c$ conjecture for $k$, for all sufficiently large $n$ satisfying

$$
(n, 2(1-\varepsilon))=1,
$$

there exists a prime ideal $\mathfrak{p}$ of $k$ such that

$$
\mathfrak{p} \nmid 2(1-\varepsilon), \quad \varepsilon^{n} \equiv 1 \quad \bmod \mathfrak{p} \quad \text { and } \quad \varepsilon^{n} \not \equiv 1 \quad \bmod \mathfrak{p}^{2} .
$$

Actually: For an integer $n$ with (4) and a prime ideal $\mathfrak{p}$ of $k$ satisfying $\mathfrak{p} \mid\left(1-\varepsilon^{n}\right)$ and $\mathfrak{p} \mid 2(1-\varepsilon)$, we see that $\operatorname{ord}_{\mathfrak{p}}\left(1-\varepsilon^{n}\right) \leq C_{2}$ for some constant $C_{2}$ independent of $n$ and $\mathfrak{p}$, where $\operatorname{ord}_{\mathfrak{p}}(*)$ is the normalized additive valuation at $\mathfrak{p}$. This follows from $\left(1-\varepsilon^{n}\right) /(1-\varepsilon) \equiv n \bmod (1-\varepsilon)$ and $(n, 1-\varepsilon)=1$ for $\mathfrak{p}$ with $\mathfrak{p} \mid(1-\varepsilon)$ and from $2 \nmid n$ for $\mathfrak{p}$ with $\mathfrak{p} \mid 2$. Therefore, by (2), for all sufficiently large $n$ with (4), there exists a prime ideal $\mathfrak{p}$ such that $\varepsilon^{n} \equiv 1 \bmod \mathfrak{p}$ and $\mathfrak{p} \nmid 2(1-\varepsilon)$. Assume that there are infinitely many $n$ with (4) such that $\varepsilon^{n} \equiv 1 \bmod \mathfrak{p}^{2}$ for all $\mathfrak{p}$ satisfying $\varepsilon^{n} \equiv 1$ $\bmod \mathfrak{p}$ and $\mathfrak{p} \nmid 2(1-\varepsilon)$. For these $n$, we have

$$
\prod_{\mathfrak{p} \mid\left(1-\varepsilon^{n}\right)}^{\prime} N \mathfrak{p} \leq\left|N\left(1-\varepsilon^{n}\right)\right|^{1 / 2} .
$$

Combining this inequality with (3), we obtain

$$
\left|N\left(1-\varepsilon^{n}\right)\right| \leq C_{1}\left|N\left(1-\varepsilon^{n}\right)\right|^{3 / 4} .
$$

This is a contradiction since the last inequality holds only for a finite number of $n$ because of (2), and hence, Claim 2 is proved.

Let $n_{1}$ and $n_{2}$ be (sufficiently large) integers satisfying (4) and $\left(n_{1}, n_{2}\right)=1$, and let $\mathfrak{p}_{i}$ be a prime ideal of $k$ satisfying $(5)_{n}$ with $n=n_{i}(i=1,2)$. Assume $\mathfrak{p}_{1}=\mathfrak{p}_{2}(:=\mathfrak{p})$. Then, from $\varepsilon^{n_{i}} \equiv 1 \bmod \mathfrak{p}$ and $\left(n_{1}, n_{2}\right)=1$, we have $\varepsilon \equiv 1$ $\bmod \mathfrak{p}$, contradicting $(5)_{n}$. Thus, we must have $\mathfrak{p}_{1} \neq \mathfrak{p}_{2}$. Therefore, by Claims 1,2 and Lemma 4, we see that there exist infinitely many prime ideals $\mathfrak{p}$ of $k$ for which $p=\mathfrak{p} \cap \mathbb{Q}$ splits completely in $k$ and

$$
\varepsilon^{N \mathfrak{p}-1}=\varepsilon^{p-1} \not \equiv 1 \quad \bmod \mathfrak{p}^{2} .
$$

Let $\mathfrak{p}$ be a prime ideal of $k$ satisfying the above two conditions. We may well assume that $p=\mathfrak{p} \cap \mathbb{Q}$ is so large that

$$
p \nmid[k: \mathbb{Q}] \cdot d_{k} \cdot h_{k},
$$

where $d_{k}$ (resp. $h_{k}$ ) is the discriminant (resp. the class number) of $k$. By (6) (and $p \nmid[k: \mathbb{Q}])$, there exists a nontrivial $\mathbb{Q}_{p}$-character $\Psi$ of $\Delta$ such that $\left(\varepsilon^{p-1}\right)^{e_{\Psi}} \not \equiv 1$ 
$\bmod \mathfrak{p}^{2}$. Let $\psi$ be, as before, an irreducible component of $\Psi$ over $\overline{\mathbb{Q}}_{p}$. Then, by $p \nmid d_{k}$, the conductor of the dual character $\psi^{*}$ of $\psi$ is divisible by $p$, and hence $\psi^{*}(p) \neq 1$. Therefore, we have $\mathcal{U}(\Psi)=\mathcal{E}(\Psi)$ by Lemma 3. Now, we obtain $\lambda_{p}(\Psi)=\mu_{p}(\Psi)=0$ from Lemma 2 and $p \nmid h_{k}$. Further, in the case (B) (= the real quadratic case), we have $\lambda_{p}=\lambda_{p}(\Psi)+\lambda_{p}\left(\Psi_{0}\right)=0$. Thus, we have proved Theorems 1 and 2 .

Remark 1. Lang [L], p. 41, presents an argument which derives the existence of infinitely many primes $p$ with $2^{p-1} \not \equiv 1 \bmod p^{2}$ from the $a b c$ conjecture for $\mathbb{Q}$. In the above proof of Theorems 1 and 2, we have used this classical argument.

Remark 2. In the above proof of Theorems 1 and 2, the existence of a unit $\varepsilon$ with $N \varepsilon=-1$ is quite essential. The author could not handle a real quadratic field whose fundamental unit has norm 1 by the method in this note.

\section{REMARK}

Let $k / \mathbb{Q}$ be a real abelian extension with $k \neq \mathbb{Q}$ and $\psi$ a fixed nontrivial homomorphism from $\Delta=\operatorname{Gal}(k / \mathbb{Q})$ to $\overline{\mathbb{Q}}^{\times}$, where $\overline{\mathbb{Q}}$ is an algebraic closure of $\mathbb{Q}$. Fixing an embedding of $\overline{\mathbb{Q}}$ into $\overline{\mathbb{Q}}_{p}$ for each prime $p$, we denote by $\Psi_{p}$ the $\mathbb{Q}_{p}$-character of $\Delta$ for which $\psi$ is an irreducible component over $\overline{\mathbb{Q}}_{p}$. We also denote by $k_{\psi}$ the subfield of $k$ corresponding to ker $\psi$ by Galois theory.

Assume that $(\mathrm{C}) k / \mathbb{Q}$ is non-cyclic or $(\mathrm{D}) k / \mathbb{Q}$ is cyclic with $[k: \mathbb{Q}]$ a composite. In the case $(\mathrm{D})$, we further assume that $k_{\psi}=k$. Then, there exist infinitely many primes $p$ satisfying (I) $p$ splits in $k$ and (II) $\lambda_{p}\left(\Psi_{p}\right)=0$.

Actually: As is easily seen, there exist infinitely many $p$ which remain prime in $k_{\psi}$ but split in $k$ (resp. which split but not completely in $k$ ) in the case (C) (resp. (D)). For these $p$, we have $\psi(p) \neq 1$, and hence $\lambda_{p}\left(\Psi_{p}\right)=0$ if $p \nmid[k: \mathbb{Q}]$ and $p \nmid h_{k}$ by Lemma 1.

\section{ACKNOWLEDGEMENT}

The author is very grateful to Hiroki Sumida for communicating Lemma 2 and its proof to him and for kindly permitting him to include them in this note.

\section{REFERENCES}

[C] J. Coates, p-adic L-functions and Iwasawa's theory, Algebraic Number Fields (Durham Symposium, 1975; ed. by A. Fröhlich), 269-353, Academic Press, London (1977). MR $\mathbf{5 7 : 2 7 6}$

[FW] B. Ferrero and L. Washington, The Iwasawa invariant $\mu_{p}$ vanishes for abelian number fields, Ann. Math., 109 (1979), 377-395. MR 81a:12005

[FK] T. Fukuda and K. Komatsu, On $\mathbb{Z}_{p}$-extensions of real quadratic fields, J. Math. Soc. Japan, 38 (1986), 95-102. MR 87d:11081

[Gi] R. Gillard, Unités cyclotomiques, unités semi-locales et $\mathbb{Z}_{l}$-extensions II, Ann. Inst. Fourier, 29 (1979), 1-15. MR 81e:12005b

[Gr] R. Greenberg, On the Iwasawa invariants of totally real number fields, Amer. J. Math., 98 (1976), 263-284. MR 53:5529

[IS] H. Ichimura and H. Sumida, On the Iwasawa invariants of certain real abelian fields II, International J. Math., 7 (1996), 721-744. CMP 97:03

[Iw] K. Iwasawa, On $\mathbb{Z}_{l}$-extensions of algebraic number fields, Ann. Math., 98 (1973), 246-326. MR 50:2120

[L] S. Lang, Old and new conjectured diophantine inequalities, Bull. AMS, 23 (1990), 37-75. MR 90k:11032 
[V] P. Vojta, Diophantine Approximations and Value Distribution Theory, Lecture Notes in Math., vol. 1239, Springer-Verlag, Berlin and New York, 1987. MR 91k:11049

[W] L. Washington, Introduction to Cyclotomic Fields, Grad. Texts in Math. no. 83, SpringerVerlag, Berlin and New York, 1982. MR 85g:11001

Department of Mathematics, Yokohama City University, 22-2, Seto, Kanazawa-ku, YOKOHAMA, 236 JAPAN

E-mail address: ichimura@yokohama-cu.ac.jp 